\title{
WHICH ONE IS THE MOST LIMITED MOVEMENT IN RESTRICTED SHOULDER JOINT
}

( ) University School of Physical Education in Wroclaw

original paper

DOI: https://doi.org/10.5114/hm.2019.83220

\section{TULAY CEVIK SALDIRAN ${ }^{1}$, OZLEM YILMAZ ${ }^{2}$}

${ }^{1}$ Istanbul Okan University, Faculty of Health Sciences, Department of Physical Therapy and Rehabilitation, Istanbul, Turkey

${ }^{2}$ Haliç University, School of Health Sciences, Department of Physiotherapy and Rehabilitation, Istanbul, Turkey

\section{ABSTRACT}

Purpose. The purpose of the study was to determine the most restrictive range of motion of the shoulder joint before treatment, as well as to examine which range of motion makes the treatment most difficult during physical therapy.

Methods. Overall, 35 patients of a physical therapy and rehabilitation clinic who met the inclusion criteria were involved in the study. Active range of motion was measured and recorded by physiotherapists using a universal goniometer in standard positions. Functional range of motion was evaluated with the Constant-Murley Score (CMS) sub-parameters. The participants were given standard physical therapy routinely performed in the clinic, 3 sessions per week for 5 weeks. At the end of the treatment, the range of motion assessments were repeated.

Results. Pre-treatment percentages were $43.24 \%$ for external rotation, $43.78 \%$ for internal rotation, $51.70 \%$ for abduction, $67.73 \%$ for flexion, $85.52 \%$ for extension. Post-treatment percentages were $62.50 \%$ for internal rotation, $72.54 \%$ for external rotation, $77.89 \%$ for abduction, $89.73 \%$ for flexion, $99.49 \%$ for extension. After treatment, the mean values of CMS subparameters were $9.71 \pm 0.86$ for flexion, $9.60 \pm 0.95$ for abduction, $8.57 \pm 1.91$ for external rotation, and $6.91 \pm 2.13$ for internal rotation. Pre- and post-treatment differences were observed to be statistically significant for the variables in the CMS $(p<0.05)$.

Conclusions. The study showed that early recovery of rotational movements, especially the internal rotation movement, at the beginning of the treatment positively affected the treatment process.

Key words: shoulder joint, internal rotation, limitation, physiotherapy

\section{Introduction}

Shoulder problems were reported to be in the third place among musculoskeletal disorders; their prevalence in the general population is $6-26 \%$ and turns out much higher when assessed by occupational risk factors [1]. The most mobile joint of the human body is the shoulder joint; its mechanism helps adjust the position of the hand in front of the body [2]. Because of the synergistic movement structure of the shoulder joint, loss of any range of motion will also affect movements in other planes [3]. Pathological tables arise owing to the equilibrium loss between mobility and functional stability. The most common pathologies that cause limitation of shoulder movement are frozen shoulder, rotator cuff rupture, and subacromial impingement syndrome, also known as impingement syndrome. Intrinsic and extrinsic mechanisms are indicated to take part in the aetiology. Vascular and mechanical factors, which complement each other, play a role in their pathophysiology [4-7].

Frieman et al. [8] reported that there were no standardization of the medical or conservative treatment program to improve shoulder movement limitations. It has been reported that in the immobilized extremity, there was a loss of motion range and muscle strength, especially related to pain factor [5]. It is known that the limitations of rotational movements in scapular posterior and anterior plane activities significantly affect the functionality of the patients [5, 7, 9]. There

Correspondence address: Tülay Çevik Saldıran, Okan University Tuzla Campus, 34959 Akfırat - Tuzla, Istanbul, Turkey, e-mail: tlyfztcvk@gmail.com

Received: July 25, 2018

Accepted for publication: January 5, 2019

Citation: Saldiran TC, Yilmaz O. Which one is the most limited movement in restricted shoulder joint. Hum Mov. 2019; 20(2):72-78; doi: https://doi.org/10.5114/hm.2019.83220. 
is reduction or dysfunction of the humeral head translation in translatoric joint play, and the main cause of movement limitation is capsule thickening accompanied by muscle strength imbalances $[10,11]$.

Shoulder joint is considerably challenging in the rehabilitation period for clinicians. It is the primary goal of healthcare professionals to bring patients to their functional capacities as soon as possible within the scope of the rehabilitation. Knowing the toughest steps in this direction is also the most helpful factor in creating the most effective treatment. In the light of this information, the purpose of this study was to determine the range of motion which is the most restrictive of the shoulder joint before treatment, as well as to examine which range of motion makes the treatment most difficult during and after physical therapy.

\section{Material and methods}

\section{Participants}

Overall, 50 volunteers who met the inclusion criteria in the examination performed by a physician and who had limited shoulder movements were evaluated in the rehabilitation department. Individuals were excluded from the study whose movement limitations were found insufficient to be included in the study $(n=9)$, who had limitations after mastectomy $(n=1)$, who had cardiac surgery history $(n=1)$, who could not participate in the exercises because of hypertension $(n=3)$, and who could not maintain continuity $(n=1)$. Eventually, the study was conducted among 35 participants. Individuals with rheumatic tables such as resulting from osteoarthritis or rheumatoid arthritis, with recurrent dislocations of shoulder joint, with infection table around the shoulder, with ischemic heart disease, with shoulder or neck surgery history, with malignancy, dementia, or cardiac pacemaker, with pain developed with radiculopathy and limitations related to muscle strength losses, with limitations of movements that became evident with neu- rological disorders, with limitations of movement after myocardial infarction, with movement loss that arouse after operations, or with complete rupture were not included in the study. Those with active range of motion if reasons of exclusion were eliminated, who had limitation of shoulder joint movement in any direction were included. Demographic information of the participants was obtained.

\section{Procedures}

Two different evaluation methods were used in our study. Goniometric measurements were applied to evaluate the range of active motion in the patients' motion planes. Active range of motion (AROM) (flexion, extension, abduction, internal rotation, external rotation) was measured and recorded by physiotherapists using a universal goniometer in standard positions [12]. Shoulder joint ranges of flexion and abduction motion were assessed in the neutral supine position with arms resting near the body. In the supine position, internal rotation and external rotation measurements were obtained with the arm at $90^{\circ}$ abduction and elbow at $90^{\circ}$ flexion supination forearm position. At the time of rotation assessment, scapular stabilization was performed by fixing the acromion with help. Attention was given so that the arm did not enter adduction and the elbow did not enter flexion. The positioning of the participants who could not make active shoulder $90^{\circ}$ abduction was carried out by the therapist. Shoulder joint extension angle was evaluated in the prone position, the arms beside the body, with no compensation.

Constant-Murley Score (CMS) was used to evaluate the patients' upper extremity functionality. The functional range of motion was detected with the CMS functional range of motion sub-parameters [13], i.e. forward flexion, lateral elevation (abduction), external and internal rotation. In the CMS evaluation, all movements were carried out while the participants were in the neutral standing position; the subjects were

Table 1. The Constant-Murley Score functional range of motion sub-parameters

\begin{tabular}{lccc}
\hline Flexion, abduction & External rotation & Internal rotation & Scoring \\
\hline $0-30^{\circ}$ & Hand behind head, elbow forward & Lateral thigh & 0 \\
$31-60^{\circ}$ & Hand behind head, elbow back & Buttock & 2 \\
$61-90^{\circ}$ & Hand to top of head, elbow forward & Lumbosacral junction & 4 \\
$91-120^{\circ}$ & Hand to top of head, elbow back & Waist (L3) & 6 \\
$121-150^{\circ}$ & Full elevation & T12 vertebra & 8 \\
$151-180^{\circ}$ & & Interscapular (T7) & 10 \\
\hline
\end{tabular}

Compiled from: http://www.orthopaedicscore.com/scorepages/constant_shoulder_score.html 
shown the required action and asked to perform it themselves bilaterally. Limitations of the movement of the affected side were evaluated with CMS (Table 1).

During the evaluation of the rotational movements, the participants were asked to touch their hand to a designated spot. For each movement performed, scoring was performed in accordance with the values in the scoring table. In the assessment of the range of motion, the sum for each sub-parameter is 10 points, and it is evaluated over the total of 40 points [14]. The participants were given the standard physical therapy routinely applied in the clinic, in continuous mode with hot pack for $30 \mathrm{~min} / \mathrm{session}$, transcutaneous electrical nerve stimulation (TENS) for $30 \mathrm{~min} / \mathrm{session}$, and ultrasound for $8 \mathrm{~min} / \mathrm{session} 1.5 \mathrm{~W} / \mathrm{cm}^{2}$, full contact technique, as 3 sessions per week and for 5 weeks. At the beginning of the treatment, the patients were taught shoulder exercises, which were performed under the supervision of physiotherapist. At the end of each session, cold pack was applied for 15 min [15]. The home exercise program was taught in detail and prescribed to each participant, accompanied by an exercise brochure. At the end of the treatment, the range of motion evaluations were repeated.

\section{Data analysis}

In the biostatistical analysis of the study, the examined variables were defined as mean \pm standard deviation $(\bar{x} \pm S D)$, frequency and percentage values. Treatment efficiency was determined with the Wilcoxon test in intra-group analysis after the treatment. The limit of significance was assumed as $p<0.05$ in the interpretations. The percentage of recovery level was calculated with the formula of AROM $\times 100 /$ normal AROM for each range of motion in order to determine the recovery levels of the standard range of motion in the participants with mean shoulder joint AROM values. The percentage of AROM before and after treatment was determined. (Normal AROM values were assumed as: $180^{\circ}$ for flexion, $180^{\circ}$ for abduction, $90^{\circ}$ for internal rotation, $90^{\circ}$ for external rotation, $45^{\circ}$ for extension). The IBM SPSS statistics version 22 software was used for biostatistical analysis.

\section{Ethical approval}

The research related to human use has been complied with all the relevant national regulations and institutional policies, has followed the tenets of the Declaration of Helsinki, and has been approved by the Istanbul Medipol University Health Research Ethics Board (number 10840098).

\section{Informed consent}

Informed consent has been obtained from all individuals included in this study.

\section{Results}

In the study, 23 (65.7\%) participants were female and $12(34.3 \%)$ were male. The right side was affected in $20(57.1 \%)$ patients and the left side in 15 (42.9\%). The subjects, with a mean age of $52.23 \pm 7.99$ years, were observed to have had complaints for $5.01 \pm 3.02$ months; their mean body mass index equalled 28.24 \pm 3.41 . Ten participants $(28.6 \%)$ had frozen shoulder, $12(34.3 \%)$ had subacromial impingement syndrome, and $13(37.1 \%)$ had rotator cuff pathology. The preand post-treatment AROM goniometric mean results are shown in Table 2 . The pre- and post-treatment differences were observed to be statistically significant $(p<0.05)$ for all variables as a result of comparing the mean measurement of the AROM of the participants' affected shoulder joints.

When the difference between the pre- and posttreatment means of AROM of the shoulder joint was examined, the most significant mean increase in the range of motion was found in abduction $\left(47.14^{\circ}\right)$, then in flexion $\left(39.60^{\circ}\right)$, external rotation $\left(26.37^{\circ}\right)$, internal rotation $\left(16.91^{\circ}\right)$, and extension $\left(6.29^{\circ}\right)$. Table 3 shows the percentage of recovery level of the shoulder joint AROM.

Table 2. Pre- and post-treatment AROM goniometric mean results $(\bar{x} \pm S D)$

\begin{tabular}{lcccc}
\hline Movement & Pre-treatment AROM & Post-treatment AROM & Differences & $p$ \\
\hline Flexion & $121.91 \pm 17.76$ & $161.51 \pm 12.72$ & $39.60 \pm 20.31$ & $<0.05$ \\
Abduction & $93.06 \pm 23.18$ & $140.20 \pm 19.68$ & $47.14 \pm 23.89$ & $<0.05$ \\
Internal rotation & $39.40 \pm 17.91$ & $56.31 \pm 15.23$ & $16.91 \pm 14.51$ & $<0.05$ \\
External rotation & $38.91 \pm 15.80$ & $65.29 \pm 15.52$ & $26.37 \pm 15.94$ & $<0.05$ \\
Extension & $38.49 \pm 7.37$ & $44.77 \pm 6.49$ & $6.29 \pm 5.82$ & $<0.05$ \\
\hline
\end{tabular}

AROM - active range of motion 
Table 3. Percentage of recovery level of the shoulder joint $\operatorname{AROM}(\bar{x} \pm S D)$

\begin{tabular}{lccc}
\hline Movement & $\begin{array}{c}\text { Pre-treatment } \\
\text { PAROMBT }\end{array}$ & $\begin{array}{c}\text { Post-treatment } \\
\text { PAROMAT }\end{array}$ & $p$ \\
\hline Flexion & 67.73 & 89.73 & $<0.05$ \\
Abduction & 51.70 & 77.89 & $<0.05$ \\
Internal rotation & 43.78 & 62.57 & $<0.05$ \\
External rotation & 43.24 & 72.54 & $<0.05$ \\
Extension & 85.52 & 99.49 & $<0.05$ \\
\hline
\end{tabular}

AROM - active range of motion, PAROMBT - percentage of active range of motion before treatment, PAROMAT percentage of active range of motion after treatment

Table 4. The mean results of CMS sub-parameters $(\bar{x} \pm S D)$

\begin{tabular}{lccc}
\hline Sub-parameter & $\begin{array}{c}\text { Pre- } \\
\text { treatment }\end{array}$ & $\begin{array}{c}\text { Post- } \\
\text { treatment }\end{array}$ & $p$ \\
\hline Elevation-flexion & $7.20 \pm 1.76$ & $9.71 \pm 0.86$ & $<0.05$ \\
Elevation-abduction & $6.80 \pm 1.95$ & $9.60 \pm 0.95$ & $<0.05$ \\
External rotation & $3.94 \pm 2.72$ & $8.57 \pm 1.91<0.05$ \\
Internal rotation & $3.49 \pm 2.19$ & $6.91 \pm 2.13<0.05$ \\
\hline
\end{tabular}

CMS - Constant-Murley Score

According to these percentages, while the most limited range of motion was the external rotation before treatment, the internal rotation movement was the most limited range of motion after treatment. The results for CMS sub-parameters are shown in Table 4.

Pre- and post-treatment differences were observed to be statistically significant for the variables in the CMS functional AROM sub-parameters $(p<0.05)$. As shown with the mean results of CMS sub-parameters of functional range of motion, while the maximum pre- and post-treatment range of motion referred to flexion, the most restricted motion was internal rotation.

\section{Discussion}

In accordance with the goniometric evaluation results, while the AROM with the greatest limitation before treatment referred to external rotation, the motion with greatest limitation after treatment was internal rotation. The CMS functional range of motion sub-parameter results showed that the most restrictive pre- and post-treatment AROM concerned internal rotation. In the literature, it has been reported that posterior capsule thickening is the leading cause of internal rotation limitation $[4,16,17]$. Thomas et al. [3] found that the thickening of the posterior capsule was higher at the dominant side than at the non-dominant side in healthy subjects. They also noted that there was a negative correlation between posterior capsule thickening and internal rotation movement and a positive correlation between posterior capsule thickening and external rotation movement. At the same time, posterior capsule thickening and glenohumeral abduction at $60^{\circ}-90^{\circ}-120^{\circ}$ showed positive correlation with superior translation of the scapula. It was also mentioned that approximation of joint segments increased compressive forces in the subacromial tissue [3].

Humerus head has been shown to be the major factor causing limitation of movement in the shoulder arm complex in many pathologies, such as anteriorsuperior translation, impingement, rotator cuff ruptures and instability. It has been reported that the limitations of the range of motion may develop as a result of ligament dysfunctions, such as hypertrophy of the coracoacromial ligament, or owing to traction osteophyte formed in the acromion [6]. The limitation of movement changed the kinematics of the glenohumeral joint, and some changes in the muscle activations over time with scapular kinematics were also observed. Serratus anterior muscle weakness, increase in upper trapezius activation, and pectoralis minor shortening have been proved to be the main factors causing limitation of movement [9]. Hung et al. [11] stated in their clinical study that muscle spasm affected the shoulder joint range of motion and that posterior deltoid, infraspinatus, and teres minor muscle spasm caused limitation of internal rotation. According to Poser and Casonato [18], even though the main factor causing internal rotation limitation is the presence of posterior capsule thickening, muscle contractures are also effective. In their case study they showed that $20^{\circ}$ internal rotation could be obtained with a single session of massage therapy on infraspinatus and teres minor muscles. They mentioned that the cause of joint limitation could not be distinguished by definite lines whether they originated from posterior capsule or infraspinatus and teres minor muscle [18].

I has also been reported that posterior capsule thickening increases the impingement formation and the risk of rotator cuff pathologies formation by affecting abnormal scapular mobility, generally anterior scapular tilt and rotational and humeral translation with anterior positioning of humeral head [4, 9, 17]. Most of the studies on shoulder joint movement limitations are focused on overhead shooting sports branches and imply that dominant shoulder range of external rotation is higher than the range of internal rotation $[16,17]$. 
T.C. Saldiran, O. Yilmaz, Physiotherapy in shoulder joint

There are studies indicating that the glenohumeral joint posterior capsule thickening and the limitations of internal rotation are not only due to posterior capsule thickening. They report that this condition develops under capsule tension with overhead recurrent activities as well as osseous adaptation [16]. However, Carcia et al. [19] found in their clinical study that the glenohumeral range of internal rotation was higher at the non-dominant side when goniometric measurements were taken at the side-lying position in healthy participants. In a limited number of studies investigating the possibility of the same mechanism also occurring in the populations outside of the overhead shooters or basketball players, researchers have reported that the tension stress on the glenohumeral joint posterior capsule may cause capsule thickening by creating fibroblast structural change, by reducing the extracellular matrix compliance. The main factors of this mechanism were shown in cadaver studies. In a cadaver study by Harryman et al. [20], the head of the humerus performed anterior and superior translation after the posterior capsule of the glenohumeral joint was sutured. This translatoric movement is predicted to create a limitation in the shoulder joint. Moreover, Dashottar and Borstad [21], moved the joint capsule to the natural contracture by $20 \%$ and $40 \%$ by coagulating the collagen tissue with a radiofrequency thermal energy probe. The pressure affecting subacromial area and humeral head translation changes during arm elevation were calculated. Limitation of movement and increase in the pressure on the subacromial area were observed with humeral head anterior and superior translation.

In the light of this information and the results of our study, we suggest that anterior glide and posterior glide of humeral head in the internal rotation of the glenohumeral joint have an effect on the internal rotation, being the last range of motion to be improved. A hypothesis came to our minds saying that the changing capsule structure causes posterior thickening and the humeral head to be anteriorly positioned and irritates the structures in the anterior compartment of the joint. We maintain that the intra-articular area will reach the end of the internal rotation range owing to the anteriorly positioned humeral head. The limitation of internal rotation is the loss of motion in the beginning of the range, not at its end. For this reason, it is possible that internal rotation motion loss was observed.

In accordance with the goniometric evaluation results of the shoulder joint before treatment in the presented study, the most limited range of motion con- cerned external rotation. James Cyriax defined the glenohumeral joint capsule pattern as external rotation range limitation being greater than abduction, and abduction being greater than internal rotation [22]. However, Rundquist and Ludewig [23] obtained different results than Cyriax in participants with idiopathic shoulder movement restriction. They showed that internal rotation limitation was greater than abduction and abduction was greater than external limitation by $56 \%$, and that the internal rotation limitation with the abduction of the affected extremity was the highest, with $92 \%$. In a cadaveric study of rotator muscle imbalance, which leads to internal impingement formation and superior labrum degeneration, it was indicated that the maximum range of external rotation motion increased with a decrease in subscapularis muscle strength, and glenohumeral contact pressure also increased. This condition has been indicated to be involved in the development of many shoulder pathologies and to provide the basis for the formation of anterior subluxations [24].

In the light of all the information listed above, in accordance with clinical observation, posterior glide and anterior glide of humeral head are performed in the external rotation motion. When we make some assumptions about the limitations of range of external rotation motion, we can say that the joint posterior compartment will not allow the limitation caused by pathology, the deficiency due to increased tension stress on the external rotator muscles, the posterior glide in the external rotation movement of the humerus head. It is possible that the glenohumeral joint exhibits agonist, antagonist movement limitation of both internal rotation and external rotation. In the glenohumeral joint, rotational movements are needed to allow the elevations to reach the full. The loss in rotational movements will affect the range of elevation. The limitation level will vary according to the size of the pathology. Loss of scapula-humeral rhythm developing with muscle imbalance will be another factor affecting the pathology process [7].

In accordance with the study outputs, as a result of the comparison of active measured averages of affected shoulder joint ranges of motion before and after treatment, it was observed that the physiotherapeutic interventions that we applied in the study content improved the joint range of motion. It has been reported that physiotherapeutic interventions should be included from the starting point of treatment in order to restore muscle strength imbalance caused by the pathology table in the restricted shoulder joint, to restore painless, stable, normal function of the shoulder joint. In 
a study investigating the efficiency of physiotherapy practices in subacromial impingement syndrome, it was reported that exercise therapy was the most effective treatment modality to overcome the loss of functional range in long and short term. Joint mobilization combined with exercise has also been shown more effective than therapeutic exercise only [25]. In research investigating the efficiency of physiotherapeutic practices in individuals with adhesive capsulitis, therapeutic exercises and joint mobilization have shown a powerful effect in gaining joint range of motion and function development. However, studies present no conclusion about the standardization of physiotherapeutic practices and exercise programs [26].

In clinical research on recovering the loss of shoulder joint range of internal rotation, an attempt to improve the limitation was taken with different stretching exercises applied to the posterior capsule of the shoulder joint. Researches indicated that both techniques that they performed were effective [27, 28]. Moore et al. [29] observed rapid improvement in basketball players with internal rotation deficit in the glenohumeral joint when they applied muscle energy techniques to increase the angle of glenohumeral horizontal adduction and internal rotation. The findings of our study show that early recovery of rotational movements, especially internal rotation, from the beginning of the treatment positively affects the treatment process. According to our clinical study results, physiotherapy practices effectively improve shoulder movement limitation and functional range of motion in a short time.

We observed that the range of motion with the highest limitation was external rotation before treatment and internal rotation after treatment. The CMS subparameter results proved that the most restricted movement was internal rotation both before and after treatment. Our findings suggest that early recovery of restricted internal rotation movement with posterior capsule stretching exercises from the beginning of treatment will positively affect the treatment process. The efficacy of massage application or muscle energy techniques to overcome the existing internal rotation limitation should be investigated.

As for the limitations of our study, it would probably be more accurate to examine the limitations of movement due to a single pathology as this would give more detailed results. It is thought that the longterm effects of short-term outcomes of rehabilitation practices should be examined in larger populations.

\section{Acknowledgments}

This research received no specific grant from any funding agency in the public, commercial, or not-forprofit sectors.

\section{Disclosure statement}

No author has any financial interest or received any financial benefit from this research.

\section{Conflict of interest}

The authors state no conflict of interest.

\section{References}

1. Van Rijn RM, Huisstede BM, Koes BW, Burdorf A. Associations between work-related factors and specific disorders of the shoulder - a systematic review of the literature. Scand J Work Environ Health. 2010;36(3): 189-201; doi: 10.5271/sjweh.2895.

2. Neviaser AS, Hannafin JA. Adhesive capsulitis: a review of current treatment. Am J Sports Med. 2010;38(11): 2346-2356; doi: 10.1177/0363546509348048.

3. Thomas SJ, Swanik KA, Swanik CB, Kelly JD. Internal rotation and scapular position differences: a comparison of collegiate and high school baseball players. J Athl Train. 2010;45(1):44-50; doi: 10.4085/10626050-45.1.44.

4. Ludewig PM, Reynolds JF. The association of scapular kinematics and glenohumeral joint pathologies. J Orthop Sports Phys Ther. 2009;39(2):90-104; doi: 10.2519/ jospt.2009.2808.

5. McClure PW, Michener LA, Karduna AR. Shoulder function and 3-dimensional scapular kinematics in people with and without shoulder impingement syndrome. Phys Ther. 2006;86(8):1075-1090; doi: 10.1093/ $\mathrm{ptj} / 86.8 .1075$.

6. Murray IR, Goudie EB, Petrigliano FA, Robinson CM. Functional anatomy and biomechanics of shoulder stability in the athlete. Clin Sports Med. 2013;32(4): 607-624; doi: 10.1016/j.csm.2013.07.001.

7. Page P. Shoulder muscle imbalance and subacromial impingement syndrome in overhead athletes. Int J Sports Phys Ther. 2011;6(1):51-58.

8. Frieman BG, Albert TJ, Fenlin JM Jr. Rotator cuff disease: a review of diagnosis, pathophysiology, and current trends in treatment. Arch Phys Med Rehabil. 1994;75(5):604-609.

9. Kibler BW, Sciascia A, Wilkes T. Scapular dyskinesis and its relation to shoulder injury. J Am Acad Orthop Surg. 2012;20(6):364-372; doi: 10.5435/JAAOS-2006-364.

10. Brantingham JW, Cassa TK, Bonnefin D, Jensen M, Globe G, Hicks M, et al. Manipulative therapy for shoulder pain and disorders: expansion of a systematic review. J Manipulative Physiol Ther. 2011;34(5):314346; doi: 10.1016/j.jmpt.2011.04.002. 
11. Hung C-J, Hsieh C-L, Yang P-L, Lin J-J. Relationship between posterior shoulder muscle stiffness and rotation in patients with stiff shoulder. J Rehabil Med. 2010;42(3):216-220; doi: 10.2340/16501977-0504.

12. Muir SW, Corea CL, Beaupre L. Evaluating change in clinical status: reliability and measures of agreement for the assessment of glenohumeral range of motion. N Am J Sports Phys Ther. 2010;5(3):98-110.

13. Roy J-S, MacDermid JC, Woodhouse LJ. A systematic review of the psychometric properties of the Constant-Murley score. J Shoulder Elbow Surg. 2010;19(1): 157-164; doi: 10.1016/j.jse.2009.04.008.

14. Çelik D. Turkish version of the modified ConstantMurley score and standardized test protocol: reliability and validity. Acta Orthop Traumatol Turc. 2016; 50(1):69-75; doi: 10.3944/AOTT.2016.14.0354.

15. Donatelli RA. Physical therapy of the shoulder. St. Louis: Churchill Livingstone; 2011.

16. Spigelman T. Identifying and assessing glenohumeral internal-rotation deficit. Athl Ther Today. 2006;11(3): 29-31; doi: 10.1123/att.11.3.23.

17. Kibler WB, Sciascia A, Thomas SJ. Glenohumeral internal rotation deficit: pathogenesis and response to acute throwing. Sports Med Arthrosc Rev. 2012;20(1): 34-38; doi: 10.1097/JSA.0b013e318244853e.

18. Poser A, Casonato O. Posterior glenohumeral stiffness: capsular or muscular problem? A case report. Man Ther. 2008;13(2):165-170; doi: 10.1016/j.math.2007. 07.002 .

19. Carcia CR, Cacolice PA, Scibek JS. Sidelying glenohumeral passive internal rotation range of motion values in a healthy collegiate population. Int J Sports Phys Ther. 2013;8(6):793-799.

20. Harryman DT $2^{\text {nd }}$, Sidles JA, Clark JM, McQuade KJ, Gibb TD, Matsen FA $3^{\text {rd }}$. Translation of the humeral head on the glenoid with passive glenohumeral motion. J Bone Joint Surg Am. 1990;72(9):1334-1343; doi: 10.2106/00004623-199072090-00009.

21. Dashottar A, Borstad J. Posterior glenohumeral joint capsule contracture. Shoulder Elbow. 2012;4(4):230 236; doi: 10.1111/j.1758-5740.2012.00180.x.

22. Noten S, Meeus M, Stassijns G, Van Glabbeek F, Verborgt O, Struyf F. Efficacy of different types of mobilization techniques in patients with primary adhesive capsulitis of the shoulder: a systematic review. Arch Phys Med Rehabil. 2016;97(5):815-825; doi: 10.1016/j. apmr.2015.07.025.

23. Rundquist PJ, Ludewig PM. Patterns of motion loss in subjects with idiopathic loss of shoulder range of motion. Clin Biomech. 2004;19(8):810-818; doi: 10.1016/ j.clinbiomech.2004.05.006.

24. Mihata T, Gates J, McGarry MH, Lee J, Kinoshita M, Lee TQ. Effect of rotator cuff muscle imbalance on forceful internal impingement and peel-back of the superior labrum: a cadaveric study. Am J Sports Med. 2009;37(11): 2222-2227; doi: 10.1177/0363546509337450.
25. Michener LA, Walsworth MK, Burnet EN. Effectiveness of rehabilitation for patients with subacromial impingement syndrome: a systematic review. J Hand Ther. 2004;17(2):152-164; doi: 10.1197/j.jht.2004.02.004.

26. Jain TK, Sharma NK. The effectiveness of physiotherapeutic interventions in treatment of frozen shoulder/ adhesive capsulitis: a systematic review. J Back Musculoskelet Rehabil. 2014;27(3):247-273; doi: 10.3233/ BMR-130443.

27. Cools AM, Johansson FR, Cagnie B, Cambier DC, Witvrouw EE. Stretching the posterior shoulder structures in subjects with internal rotation deficit: comparison of two stretching techniques. Shoulder Elbow. 2012;4(1):56-63; doi: 10.1111/j.1758-5740.2011.00159.x.

28. Manske RC, Meschke M, Porter A, Smith B, Reiman M. A randomized controlled single-blinded comparison of stretching versus stretching and joint mobilization for posterior shoulder tightness measured by internal rotation motion loss. Sports Health. 2010;2(2):94-100; doi: 10.1177/1941738109347775.

29. Moore SD, Laudner KG, McLoda TA, Shaffer MA. The immediate effects of muscle energy technique on posterior shoulder tightness: a randomized controlled trial. J Orthop Sports Phys Ther. 2011;41(6):400-407; doi: 10.2519/jospt.2011.3292. 